\title{
Frecuencia de hipermovilidad articular en escolares entre 8 y 14 años de un colegio de San Martin de Porras
}

Frequency of articular hypermobility in school children between 8 and 14 years of age in a school in the district of San Martin de Porres,

\author{
Brenda Yhomira Malca Sáciga ${ }^{1, a}$, Betsabeth Santos Ramírez ${ }^{1, a}$, Yessenia Stefani Sebastian Palomino ${ }^{1, a}$, \\ Manuel Tomás Castillo Portilla ${ }^{1, b}$, Elisa Verónica Milla Zavaleta ${ }^{1, c}$.
}

\section{RESUMEN}

Objetivos: Determinar la frecuencia de HA en escolares entre 8 y 14 años en un colegio del distrito de San Martin de Porres, y describir la HA según edad, género, índice de masa corporal (IMC) y articulaciones examinadas. Material y Métodos: Estudio descriptivo, observacional tipo transversal, incluyó 232 escolares. Al evaluar se utilizó el Beighton score para medir la HA y el goniómetro para medir rangos articulares, balanza electrónica y tallímetro para determinar el peso y la talla y así calcular el IMC. Resultados: La frecuencia de HA fue 101 escolares, 63 mujeres y 38 hombres. Según la edad la HA disminuye a excepción entre los 11 y 12 años donde no existe variación, a los 13 años la frecuencia incrementa y decrece a los 14 años. Según el IMC, 81 escolares con peso normal tienen $(45,5 \%)$ HA. La articulación con mayor HA fue el codo derecho 172. Conclusiones: La frecuencia de HA fue 101 escolares (43,53\%). Según edad fue mayor en niños de 8 años. Según género, las mujeres tienen 15\% más de HA que los varones. Según IMC la HA fue mayor en escolares con peso normal 8,5\% más y según las articulaciones examinadas fue mayor en el codo derecho $(74,1 \%)$.

PALABRAS CLAVE: Hipermovilidad articular, Beighton score, niños.

\section{SUMMARY}

Objective: To determine the frequency of AH in school children between 8 and 14 years of age in a school in the district of San Martin de Porres, and to describe the HA according to age, gender, body mass index (BMI) and joints examined. Material and Methods: A descriptive, observational cross-sectional study included 232 schoolchildren. When evaluating, the Beighton score was used to measure the HA and the goniometer to measure joint ranges, electronic scale and height meter to determine the weight and height to calculate the BMI. Results: The frequency of HA was 101 schoolchildren, 63 women and 38 men. According to age, HA decreases except between 11 and 12 years where there is no variation, at 13 years the frequency increases and decreases at 14 years. According to the IMC, 81 schoolchildren with normal weight have (45.5\%) HA. The joint with the highest HA was the right elbow 172. Conclusions: The frequency of HA was 101 schoolchildren (43.53\%). According to age, it was higher in children of 8 years. According to gender, women have $15 \%$ more HA than men. According to BMI, HA was higher in schoolchildren with normal weight $8,5 \%$ and according to the joints examined it was greater in the right elbow (74.1\%).

KEY WORDS: Joint Hypermobility, Beighton score, children.

\footnotetext{
${ }^{1}$ Escuela de Tecnología Médica, Facultad de Medicina, Universidad Peruana Cayetano Heredia. Lima, Perú.

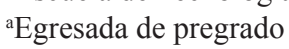

${ }^{b}$ Docente; Medico Cirujano, Magister en Epidemiologia

${ }^{c}$ Docente; Licenciada en Tecnologia Medica.
} 


\section{INTRODUCCIÓN}

La hipermovilidad articular o hiperlaxitud articular (HA) (1) se define como una condición donde la mayoría de las articulaciones sinoviales de las personas tienen un rango articular excesivo considerando la edad, el género y los antecedentes étnicos de la persona (2). Las personas con HA tienen una relación anormal de colágeno tipo I y III. En relación a factores genéticos, la laxitud articular se asocia a una herencia dominante con manifestaciones fenotípicas dando como resultado defectos en los genes que codifican las proteínas de la matriz del tejido conectivo, como el colágeno de tipo I, III, la elastina y la fibrilina. A nivel bioquímico, la alteración que se produce en la laxitud articular no se conoce exactamente; se han detectado alteraciones en la estructura de las fibras de colágeno (pequeño diámetro) y una cantidad aumentada de matriz interfibrilar, elastina y fibrocitos (3).

La hipermovilidad articular genera distenciones musculo tendinosas yuxta-articulares y compresiones de la membrana sinovial, sometiendo a las superficies articulares a apoyos direccionales no fisiológicos (3), esto puede generar subluxaciones, fracturas, ruptura de ligamentos tenosinovitis, artritis temprana, discopatías, enfermedades degenerativas y osteoporosis $(4,5)$.

Cerca del $10 \%$ a $30 \%$ de la población a nivel mundial tiene hipermovilidad articular (6), aunque la mayoría no tiene síntomas, la HA ha demostrado ser una de las principales causas de dolor crónico. Sin embargo, muchas personas con HA permanecen sin diagnosticar (7-9).

En el Reino Unido y Holanda, el porcentaje de HA fue de $19,2 \%$ en niños de 14 años y $26 \%$ en niños de 6 a 12 años respectivamente $(10,11)$, en España, se obtuvo un porcentaje de $25,4 \%$ en escolares entre las edades de 8 y 12 (12), en China hallaron 28\% de HA en mayores de 10 años y $67 \%$ en menores de 10 años (13), en la India se obtuvo un porcentaje de $58,7 \%$ en niños de 3 a 19 años (14). El porcentaje de HA en Estados Unidos fue de 34\% (15), en Argentina 37,3\% (16), en Brasil 36\% (17) y se reportó 33\% de casos de Síndrome benigno de hiperlaxitud articular (SBHA) en Ecuador (18).

En Perú, el porcentaje de SBHA fue $28,8 \%$ en escolares entre los 6 y 16 años de edad en la ciudad de Trujillo (19), por otro lado, en un estudio realizado en el Callao resultó que en niños menores de 8 años la prevalencia del SBHA se aproxima al 50\% (20). En el 2005, Socca S y Mendoza J encontraron un 72,2\% de SBHA en Lima y demuestra que existe un mayor porcentaje en las niñas con $50,8 \%$ que en los niños entre los 7 y 8 años sobre todo a los 7 años con $61,3 \%$ (21).

El presente trabajo se analizaron niños y adolescentes que tienen hipermovilidad articular, empleando el Beighton score junto con el goniómetro, siendo instrumentos válidos para la detección de hipermovilidad articular obteniendo medidas más exactas y objetivas evitando falsos positivos ya que el solo uso del Beighton score como evaluación clínica da resultados sobreestimados.

El objetivo del presente trabajo fue determinar la frecuencia de hipermovilidad articular en escolares entre 8 y 14 años en el colegio Parroquial San Columbano del distrito de San Martín de Porres, asi como describir la hipermovilidad articular según edad, género e índice de masa corporal (IMC) en escolares y describir la frecuencia de hipermovilidad articular según articulaciones examinadas.

\section{MATERIAL Y MÉTODOS}

El estudio se desarrolló con un diseño observacional, descriptivo de tipo transversal. La población estuvo conformada por 585 escolares siendo niños y adolescentes entre 8 y 14 años de edad, de ambos géneros del Colegio Parroquial San Columbano del distrito de San Martín de Porres. Se calculó el tamaño muestral con los siguientes parámetros: tamaño de la población 585 escolares, con una frecuencia hipotética de $52,4 \%$, nivel de confianza de $95 \%$ y un error de $5 \%$ para un tamaño de muestra de 232 escolares, basado en el estudio de Suárez, María y Gómez (22). Los resultados se obtuvieron usando la plataforma OpenEpi, versión 3 (23).

Se incluyó a escolares de ambos géneros entre las edades de 8 y 14 años quienes se encontraban matriculados en el colegio Parroquial San Columbano. Se excluyó a los escolares con lesiones músculoesqueléticas prexistentes que fueron referidos por los padres mediante la ficha de recolección de datos para padres y si durante la evaluación el escolar presentó dolor al aplicar el Beighton score.

\section{Procedimientos}

Previo a la ejecución, se realizó un análisis de confiabilidad inter-observador conformado por las investigadoras $\mathrm{y}$ un especialista de terapia física 
y rehabilitación. Se evaluaron a 5 niños y con los resultados se calculó el coeficiente de correlación intraclase obteniendo rango entre 0,55 y 0,62 de coeficiente de correlación para el Beighton score. Además, se hizo un análisis de confiabilidad antes de realizar las mediciones con el goniómetro calculando el valor de coeficiente de correlación con la prueba ANOVA no existiendo diferencia significativa entre los evaluadores encontrándose entre en un rango de 0,84 y 1 .

Obtención de permisos: Primero se coordinó con la directora del colegio para gestionar el permiso de realizar la evaluación a los escolares, luego se organizó un cronograma de actividades para la recolección de datos.

Ubicación, invitación de participantes y obtención del consentimiento informado: Posteriormente se envió los asentimientos para los escolares, consentimientos para padres y la ficha de recolección de datos para padres en los cuadernos de control. Durante los días que se programó la evaluación, los escolares trajeron los permisos firmados.

Evaluación de elegibilidad: Los escolares ingresaron al auditorio, la evaluación duró 8 minutos por cada escolar. Hubo 2 módulos: En terapia física, se utilizó el Beighton score para medir la HA y el goniómetro para medir rangos articulares de codos, rodillas y de la $5^{\circ}$ metacarpofalángica y en el módulo de nutrición se utilizó una balanza electrónica y tallímetro, teniendo como referencia la "Guía para el personal de la salud del primer nivel de atención"(24). Durante el proceso de evaluación el escolar estuvo vestido con polo, short y medias. Esta evaluación se llevó a cabo al término de las clases.

Recolección de datos: Toda la información fue registrada en una ficha de recolección de datos. Terminada la evaluación, se envió un informe sobre los resultados de los escolares a los padres de familia y al colegio, donde se programó una sesión educativa sobre nutrición y HA con una serie de ejercicios y recomendaciones, todo ello detallado en un folleto. Concluyendo con la recolección de datos, se catalogó a los escolares con HA teniendo un puntaje $\geq 4 / 9$ y se clasificó en 4 categorías el Índice de Masa Corporal (IMC) mediante las gráficas de la Organización Mundial de la Salud (OMS) (25).

Tallímetro (24): Es un instrumento que se emplea para medir la estatura de niños mayores de dos años y se mide de pie. Marca: Diseños Flores S.R.L siendo elaborado bajo especificaciones técnicas.

Balanza electrónica (24): Sirve para pesar tanto niños como adultos hasta un peso de $120 \mathrm{~kg}$. Marca: Sensun - Bluetooth Body Fat Scale Model iF912B.

La balanza electrónica y tallímetro sirvieron para determinar el peso y la talla. Se dividió el peso en kilogramos por el cuadrado de la talla en metros $(\mathrm{Kg} /$ $\mathrm{m}^{2}$ ) y así se obtuvo el IMC (26). Para categorizar el IMC pediátrico se utiliza las tablas de percentiles según la Organización Mundial de la Salud (OMS) clasificando el peso bajo con un percentil $<5$, el peso normal con un percentil 5 hasta $\leq 85$, el sobrepeso con percentil $>85$ hasta el percentil $\leq 97$ y la obesidad con un percentil $>97$ para la edad y el género (27).

Beighton score (28): Se utilizó por primera vez en África en el pueblo de Tsuana Phokeng al noroeste de Johannesburgo (29). El Gold Standard para medir la HA es el uso del Beighton score junto con el goniómetro, siendo así instrumentos válidos a nivel internacional en todas las edades, tiene una especificidad del $99,3 \%$ y sensibilidad del $0,8 \%$ (30).

Los escolares fueron valorados en una escala de 9 puntos, considerando 1 punto por cada sitio hipermóvil, realizándose en ambos hemicuerpos.

Goniómetro (31): Es el instrumento para medir los rangos articulares de las articulaciones. Se midió 3 articulaciones: codo, rodilla y la quinta metacarpofalángica en ambos hemicuerpos. Se utilizó como referencia el libro de goniometría titulado "GONIOMETRIA una herramienta para la evaluación de las incapacidades laborales" (31).

\section{Aspectos éticos}

Este proyecto fue aprobado por el Comité Institucional de Ética de la Universidad Peruana Cayetano Heredia con código SIDISI: 67513 y del Colegio Parroquial San Columbano. Se entregó un consentimiento informado a los apoderados de los participantes y un asentimiento informado a los participantes entre las edades de 8 y 14 años. Solo las investigadoras tuvimos acceso a los datos obtenidos.

Los apoderados tuvieron conocimiento si su hijo tuvo o no HA además, si su Índice de Masa Corporal (IMC) se encontró en un rango ideal. Se le informó de manera personal y confidencial los resultados 
obtenidos en ambas pruebas, para que así se pueda prevenir futuros problemas en la salud de su hijo(a). Además, se planteó una serie de ejercicios específicos para la HA y una sesión educativa sobre nutrición. Toda esta información se entregó en un folleto incluyendo recomendaciones. Para garantizar la seguridad de los niños la prueba se realizó con la supervisión de padres y/o apoderado. No se tomó fotografías o grabaciones de los niños para proteger su intimidad. Los datos obtenidos fueron utilizados solo para fines de investigación.

\section{Plan de análisis}

Al finalizar la recolección de datos, en el programa Microsoft Excel 2013 se elaboró una base de datos. Para el análisis estadístico se migró los datos al programa de Stata versión 13 (Stata Corp, Tx, USA), se calculó el promedio, desviación estándar, mediana para las variables cuantitativas y porcentajes para las variables cualitativas. Además, se calcularon los OR para las variables de género e IMC. Las variables del estudio fueron clasificadas para la elaboración de tablas de frecuencia, siendo estas; hipermovilidad articular, hipermovilidad articular de codos, hipermovilidad articular de rodillas, hipermovilidad articular de pulgar, hipermovilidad de meñiques, hipermovilidad de tronco, IMC, género, peso, talla y edad.

\section{RESULTADOS}

De acuerdo a la edad, el promedio fue 11,08 $\pm 2,10$ años. De los 232 escolares 124 evaluados $(53,45 \%)$ correspondieron al género femenino y 108 evaluados $(46,55 \%)$ al género masculino. Según el IMC, 178 escolares $(76,72 \%)$ tuvieron peso normal y 54 escolares $(23,28 \%)$ tuvieron peso no ideal. La frecuencia de HA fue 101 escolares (43,53\%) (tabla $1)$.

Tabla 1. Características generales en escolares entre 8 y 14 años de un colegio del distrito de San Martin de Porres en el 2018.

\begin{tabular}{lcc}
\hline Características generales & $\mathbf{n = 2 3 2}$ & $\mathbf{\%}$ \\
\hline Edad & $\begin{array}{c}\text { Promedio } \\
11,08\end{array}$ & $\begin{array}{c}\text { Desviación estándar } \\
\text { Género }\end{array}$ \\
Femenino & 124 & 2,10 \\
Masculino & 108 & 53,45 \\
IMC & & 46,55 \\
Peso normal & 178 & \\
Peso no ideal & 54 & 76,72 \\
Hipermovilidad Articular & & 23,28 \\
Positivo & 101 & \\
Negativo & 131 & 43,53 \\
\hline
\end{tabular}

Tabla 2. Frecuencia de hipermovilidad articular según características sociodemográficas de escolares entre 8 y 14 años de un colegio del distrito de San Martín de Porres en el 2018.

\begin{tabular}{|c|c|c|c|c|c|}
\hline \multirow{3}{*}{ Características sociodemográficas } & \multicolumn{4}{|c|}{ Hipermovilidad Articular } & \multirow{3}{*}{ OR } \\
\hline & \multicolumn{2}{|c|}{ Positivo } & \multicolumn{2}{|c|}{ Negativo } & \\
\hline & $\mathrm{n}=101$ & $\%$ & $\mathrm{n}=\mathbf{1 3 1}$ & $\%$ & \\
\hline \multicolumn{6}{|l|}{ Género } \\
\hline Femenino & 63 & $50,80 \%$ & 61 & 49,20 & \\
\hline Masculino & 38 & $35,20 \%$ & 70 & 64,80 & 1,90 \\
\hline \multicolumn{6}{|l|}{ IMC } \\
\hline Peso no ideal (bajo peso, sobrepeso y obesidad) & 20 & 37,0 & 34 & 63,0 & \\
\hline Normal & 81 & 45,50 & 97 & 54,5 & $\mathbf{0 , 7 0}$ \\
\hline
\end{tabular}


Tabla 3. Frecuencia de hipermovilidad articular según edad de escolares entre 8 y 14 años de un colegio del distrito de San Martín de Porres en el 2018.

\begin{tabular}{lllll}
\hline \multirow{2}{*}{ Edad } & \multicolumn{3}{c}{ Hipermovilidad articular } \\
\cline { 2 - 5 } & \multicolumn{2}{c}{ Positivo } & \multicolumn{2}{c}{ Negativo } \\
& $\mathbf{n}=\mathbf{1 0 1}$ & $\mathbf{\%}$ & $\mathbf{n}=\mathbf{1 3 1}$ & $\mathbf{\%}$ \\
\hline $\mathbf{8}$ años & 25 & $73,5 \%$ & 9 & $26,5 \%$ \\
$\mathbf{9}$ años & 20 & $58,8 \%$ & 14 & $41,2 \%$ \\
$\mathbf{1 0}$ años & 7 & $20,0 \%$ & 28 & $80,0 \%$ \\
$\mathbf{1 1}$ años & 7 & $31,8 \%$ & 20 & $68,2 \%$ \\
$\mathbf{1 2}$ años & 7 & $31,8 \%$ & 15 & $68,2 \%$ \\
$\mathbf{1 3}$ años & 21 & $55,3 \%$ & 17 & $44,7 \%$ \\
$\mathbf{1 4}$ años & 14 & $33,3 \%$ & 28 & $66,7 \%$ \\
\hline
\end{tabular}

Tabla 4. Frecuencia de hipermovilidad articular según articulaciones examinadas en escolares entre 8 y 14 años de un colegio del distrito de San Martín de Porres en el 2018 .

\begin{tabular}{|c|c|c|c|}
\hline \multirow{2}{*}{$\begin{array}{l}\text { Hipermovilidad } \\
\text { articular } \\
\text { en articulaciones } \\
\text { examinadas }\end{array}$} & \multicolumn{3}{|c|}{ Hipermovilidad articular } \\
\hline & Positivo & Negativo & $\%$ \\
\hline $\begin{array}{l}\text { Articulación de codo } \\
\text { derecho }\end{array}$ & 172 & 60 & 74,1 \\
\hline $\begin{array}{l}\text { Articulación de codo } \\
\text { izquierdo }\end{array}$ & 167 & 65 & 72,0 \\
\hline Pulgar derecho & 101 & 131 & 43,5 \\
\hline Pulgar Izquierdo & 101 & 131 & 43,5 \\
\hline Meñique derecho & 59 & 173 & 25,4 \\
\hline Meñique izquierdo & 76 & 156 & 32,8 \\
\hline $\begin{array}{l}\text { Articulación de } \\
\text { rodilla derecha }\end{array}$ & 90 & 142 & 38,8 \\
\hline $\begin{array}{l}\text { Articulación de } \\
\text { rodilla izquierda }\end{array}$ & 90 & 142 & 38,8 \\
\hline Tronco & 101 & 131 & 43,5 \\
\hline
\end{tabular}

Según el género las mujeres tienen 15\% más de HA que los varones, de las 124 mujeres evaluadas 63 $(50,8 \%)$ tienen HA y de los 108 varones 38 (35,2\%); teniendo un $\mathrm{OR}=1,90$ expresando que las mujeres tienen 1,9 veces más probabilidad de HA que los varones. (tabla 2 ).

En la categoría del IMC, los escolares con peso no ideal tienen menos HA, de los 54 escolares con peso no ideal 20 (37\%) tienen HA y de los 178 escolares con peso normal $81(45,5 \%)$, teniendo un $\mathrm{OR}=0,70$ expresando que los escolares con peso no ideal tienen $30 \%$ menos probabilidad de presentar HA (tabla 2).

$\mathrm{Al}$ determinar la muestra de escolares que presentan HA ( $\mathrm{n}=101)$ con la edad, se encontró que 25 escolares $(73,5 \%)$ tienen 8 años, $20(58,8 \%)$ tienen 9 años, 7 (20\%) tienen 10 años, $7(31,8 \%)$ tienen 11 años, 7 $(31,8) \%$ tienen 12 años, $21(55,3 \%)$ tienen 13 años y $14(33,3 \%)$ restante tienen 14 años (tabla 3 ).

La articulación que presentó mayor frecuencia de hipermovilidad articular fue el codo derecho con 172 $(74,10 \%)$ y la menos frecuente el meñique derecho con $59(25,40 \%)$ (tabla 4$)$.

\section{DISCUSIÓN}

En la muestra estudiada 101 escolares, que representan el 43,53\%, tuvieron HA cifra que se aproxima ligeramente a estudios realizados en países de América Latina como es el caso de Argentina (37,3\%), Brasil (36\%), Ecuador (33\%) y Colombia (52\%) $(16-18,22)$. Sin embargo, en el estudio realizado por Tesen E, Tuesta J, Alfaro P y Granados J en Perú, obtuvieron una frecuencia de 69,55\% (32), esta amplia variación probablemente sea explicable por las edades evaluadas en el referido estudio comprendidas entre los 7 y 10 años ya que la bibliografía refiere que a menor edad existe mayor frecuencia de HA (12). Además, de la diferente manera de evaluar la HA sin el uso del goniómetro.

En dos revisiones bibliográficas, la frecuencia fue mayor en mujeres que en hombres, así como lo demuestra el estudio peruano de Socca S y Mendoza $\mathrm{J}$ obteniendo $50,82 \%$ entre las edades de 7 y 8 años (21). En Colombia, el 68\% de mujeres entre 4 y 17 años tienen HA (22), en ambos estudios las cifras se aproximan a la nuestra, donde la frecuencia de HA en las mujeres fue de 62,38\%. Según Bauer, Dunne y Whitewood estos hallazgos de predominio de género se deben a cambios hormonales (33).

La HA va disminuyendo conforme aumenta la edad, excepto en edades entre 11 y 12 años donde hay una regularidad de frecuencia en 7 escolares $(31,08 \%)$, en la edad de 13 años la frecuencia de HA aumenta y luego decrece a los 14 años. En el 
2010, al realizar el Test de Beighton en España (Granada) la frecuencia de HA a los 8 años hubo un porcentaje de $24,7 \%, 9$ años $22,0 \%, 10$ años $25,2 \%$, 11 años 23,8\% y finalmente 12 años 4,3\% (12)

Con respecto al IMC obtuvimos una mayor frecuencia de HA en niños con peso normal. En el estudio de Tabaku, Klieger, Wrontniak, Sherry, ZemelB y Stettler realizado en el 2014 la prevalencia de HA fue del $14 \%$ entre las personas con obesidad y $9 \%$ entre las personas con peso saludable (34). En la India se obtuvo una prevalencia de 58,7\% de HA en niños con moderada a severa desnutrición (14).

En el presente estudio la articulación con mayor frecuencia de HA fue el codo derecho con $172(74,10 \%)$, en la revisión de la literatura no se encontró una explicación fisiológica del porque hay articulaciones con mayor predisposición a ser hipermóviles. El ciclo hormonal afecta la laxitud articular pero solo en la articulación de rodilla (35). Otros estudios encontraron hallazgos con respecto a que articulación es más hipermóvil como: la quinta articulación metacarpofalángica con $82 \%$ (22) y rodillas con 48\% (36). Concluyendo así que no existe una articulación con mayor susceptibilidad. No disponemos de información suficiente para atribuir esta heterogeneidad en las articulaciones examinadas.

$\mathrm{Al}$ realizar el estudio tuvimos ciertas limitaciones, no se halló puntos de corte para la puntuación del Beighton score según género y edad por la falta de estudios poblacionales en Latinoamérica. Además, el instrumento que utilizamos tiene una baja sensibilidad, hay gran probabilidad de detectar erróneamente, también en nuestro estudio la confiabilidad no fue óptima pero si para goniómetro ya que alcanzamos valores de 0,84. Se sugiere que en futuras investigaciones realicen estudios poblacionales para determinar los puntos de corte en la población peruana.

\section{CONCLUSIONES}

La frecuencia de HA fue 101 (43,53\%) escolares evaluados.

La frecuencia de HA según edad fue mayor a los 8 años $\mathrm{n}=25(73,5 \%)$ y menor a $\operatorname{los} 10$ años $\mathrm{n}=7$ $(20,0 \%)$.

Las mujeres tienen 15\% más de HA que los varones.
Los escolares con peso no ideal tienen $30 \%$ menos de probabilidad de presentar HA.

La articulación con mayor frecuencia de HA es el codo derecho $172(74,10 \%)$.

\section{Correspondencia:}

Manuel Castillo-Portilla

Correo electrónico: manuel.castillo@upch.pe

\section{REFERENCIAS BIBLIOGRÁFICAS}

1. Grahame R. Sindrome de Hiperlaxitud articular ¿Qué puede hacer una persona para ayudarse a sí misma? Asedh. 2006; 1:1-19.

2. Simmonds J, Keer R. Hipermobility and the hypermobility syndrome. Man Ther. 2007;12(4):298309. doi: 10.1016/j.math.2007.05.001

3. Martínez J, Suarez R, Menéndez F. El síndrome de hiperlaxitud articular en la práctica clínica diaria. Revista Cubana de Reumatología. 2013; 15(1):36-41.

4. Kirby A, Davies R. Developmental coordination disorder and joint hypermobility syndromeoverlapping disorders? Implications for research and clinical practice. Child Care, Health Dev. 2006; 32:513-519.

5. Zimmermann I. Nadmierna ruchomość stawów a choroby reumatyczne. Reumatologia. 2007; 45: 397-403.

6. Beighton P, Grahame R, Bird H. Hypermobility of Joints. New York: Springer; 2012.

7. Klemp P, Williams S, Stansfield A. Articular mobility in Maori and European New Zealanders. Rheumatology. 2002; 41:554-7.

8. Grahame R. Pain, distress, and joint hyperlaxity. Joint, bone, spine. 2000; 67:157-63.

9. Grahame R. Heritable disorders of connective tissue. Best Practice \& Research in Clinical Rheumatology. 2000:14(2):345-61.

10. Clinch J, Deere K, Sayers A, Palmer S, Riddoch C Tobias J, et al. Epidemiology of generalized joint laxity (hypermobility) in fourteen-year-old children from UK: a population- based evaluation. Arthritis Rheum. 2011;63(9):2819-27. doi: 10.1002/art.30435.

11. Van der Giessen L, Liekens D, Rutgers K, et al. Validation of Beighton score and prevalence of connective tissue signs in 773 Dutch children. J Rheumatol. 2001; 28(12):2726-30.

12. Zurita F, Ruiz L, Martínez A, Fernández M, Rodríguez C, López R. Hiperlaxitud ligamentosa (test de Beighton) en la población escolar de 8 a 12 años de la provincia de Granada. Reumatol Clin. 2010; 6(1):510.

13. Cheng J, Chan P, Hui P. Joint laxity in children. J 
Pediatr Orthop. 1991; 11(6):752-6.

14. Hasija R, Khubchandani P, Shenoi S. Joint hypermobility in Indian children. Clinical and Experimental Rheumatology. 2008; 26:146-150.

15. Arroyo I, Brewer E, Giannini E. Arthritis/arthralgia and hypermobility of the joints in schoolchildren. $\mathrm{J}$ Rheumatol. 1988; 15(6):978-80.

16. De Cunto C, Moroldo M, Liberatore D, Imach, E. Hiperlaxitud Articular: estimación de su prevalencia en niños en edad escolar. Arch argent pediatr. 2001; 99(2):105-110.

17. Feitosa K. Síndrome de hipermobilidade articular. Arq Bras Pediat. 1996; 3:105-108.

18. Torres A, González P, Villegas V, Moreno M. Hipermovilidad articular benigna en niños escolares sanos de tres escuelas fiscales de Guayaquil. Reumatología al día. 2004; 8(1):0-0.

19. Cabello E, Barrantes V. Prevalencia del síndrome benigno de hiperlaxitud articular en escolares de Trujillo. Revista Acta Médica Peruana. 1993; 17:1-2.

20. Yasuda R. Prevalencia del síndrome benigno de hiperlaxitud articular en el distrito del Callao: Tesis para optar la especialización en Medicina Física y Rehabilitación. Lima, Perú: Universidad Nacional Mayor de San Marcos; 1991.

21. Socca S, Mendoza J. Relación causal del síndrome benigno de hiperlaxitud articular y la escoliosis postural. Tesis de licenciatura. Lima, Perú: Universidad Nacional Mayor de San Marcos; 2005.

22. Suárez D, María A, Gómez M. Relación entre hipermovilidad articular generalizada y dolor articular en niños de 4 a 17 años en Pasto, Colombia. Rev Colomb Reumatol. 2015; 22(4):210-216.

23. Dean A, Sullivan K, Soe M. OpenEpi: Open Source Epidemiologic Statistics for Public Health. Public Health Rep. 2009; 124(3): 471-474.

24. Rojas M, Vargas R. La medición de la talla y el peso; guía para el personal de la salud del primer nivel de atención. Lima: Ministerio de Salud. 2004.

25. WHO Multicentre Growth Reference Study Group. WHO Child Growth Standards: Body mass index-forage: methods and development. Geneva: WHO; 2006.

Recibido: $15 / 02 / 2020$

Aceptado: 22/04/2020
26. Lodoño C, Barbosa N, Tovar G, Sánchez C. Sobrepeso en escolares: Prevalencia, factores protectores y de riesgo en Bogotá. Tesis de Maestría. Bogotá, Colombia: Universidad Colegio Mayor de Nuestra Señora del Rosario; 2009. 21 pp.

27. Hernández G. Prevalencia de sobrepeso y obesidad, y factores de riesgo, en niños de 7-12 años, en una escuela pública de Cartagena Septiembre-Octubre de 2010. Tesis de Maestría. Cartagena, Colombia: Universidad Nacional de Colombia 2011. 52 pp.

28. Smith-Engelsman B, Klerks M, Kirby A. Beighton score: A valid measure for generalized hypermobility in children. J Pediatr. 2011;158(1):119-23. doi: 10.1016/j.jpeds.2010.07.021

29. Beighton P, Solomon L, Soskolone C. Articular mobility in an African population. Ann rheum Dis. $1973 ; 32: 413$.

30. Harjodh S. Beighton scores and cut-offs across the lifespan: cross-sectional study of an Australian population. Rev Reumathology. 2017; 56:1857-1864.

31. Taboadela C. Goniometría una herramienta para la evaluación de las incapacidades laborales. Buenos Aires: Asociart Art; 2007.

32. Tesen E, Tuesta J, Alfaro P, Granados J. Frecuencia de las características de Hiperlaxitud Articular en edad escolar de 7 a 10 años. Rev Hered Rehab. 2016; 1:68-73.

33. Bauer S, Dunne B, Whitewood C. Simultaneous bilateral elbow dislocation with bilateral medial epicondyle fractures in a 13-year-old female gymnast with hyperlaxity. BMJ Case Reports. 2012;1: 1-4.

34. Bout-Tabaku S, Klieger S, Wrontniak B, Sherry D, Zemel B, Stettler N. Adolescent obesity, joint pain, and hypermobility. Pediatric Rheumatology. 2014; 12:1-5.

35. Zarzuela B, Paterno M, Myer G, Romani W, Hewett T. The effects of the menstrual cycle on anterior knee laxity: a systematic review. Sports Med. 2006; 36(10):847-862.

36. Clarck C, Simmonds J. An Exploration of the prevalence of hipermobility and joint hipermobility syndrome in omani women attending a hospital physiotherapy Service. Rev Musculoskelet Care Med. 2011; 9(1):1-10. 